OPEN ACCESS

Edited by:

Jinyong Peng,

Dalian Medical University, China

Reviewed by:

Xufeng Tao',

Dalian Medical University, China Guoxun Chen,

University of Tennessee, Knoxville,

United States

*Correspondence:

Yingjun Zhao

zhaoyingjun@fudan.edu.cn

Xianghuo $\mathrm{He}$

xhhe@fudan.edu.cn

${ }^{\dagger}$ These authors have contributed equally to this work.

Specialty section: This article was submitted to Gastrointestinal and Hepatic

Pharmacology,

a section of the journal

Frontiers in Pharmacology

Received: 21 February 2018 Accepted: 26 April 2018

Published: 15 May 2018

Citation:

Chen D, Wang H, Chen J, Li Z, Li S,

Hu Z, Huang S, Zhao $Y$ and He $X$

(2018) MicroRNA-129-5p Regulates

Glycolysis and Cell Proliferation by

Targeting the Glucose Transporter

SLC2A3 in Gastric Cancer Cells.

Front. Pharmacol. 9:502.

doi: 10.3389/fphar.2018.00502

\section{MicroRNA-129-5p Regulates Glycolysis and Cell Proliferation by Targeting the Glucose Transporter SLC2A3 in Gastric Cancer Cells}

\author{
Di Chen ${ }^{1 t}$, Hui Wang ${ }^{2 t}$, Jie Chen ${ }^{1}$, Zhe $\mathrm{Li}^{1}$, Shengli Li', Zhixiang Hu', Shenglin Huang ${ }^{1}$, \\ Yingjun $\mathrm{Zhao}^{1 *}$ and Xianghuo $\mathrm{He}^{1,3 *}$ \\ ${ }^{1}$ Fudan University Shanghai Cancer Center and Institutes of Biomedical Sciences, Shanghai Medical College, Fudan \\ University, Shanghai, China, ${ }^{2}$ State Key Laboratory of Oncogenes and Related Genes, Shanghai Cancer Institute, Renii \\ Hospital, Shanghai Jiao Tong University School of Medicine, Shanghai, China, ${ }^{3}$ Department of Oncology, Collaborative \\ Innovation Center for Cancer Medicine, Shanghai Medical College, Fudan University, Shanghai, China
}

Tumor cells increase their glucose consumption through aerobic glycolysis to manufacture the necessary biomass required for proliferation, commonly known as the Warburg effect. Accumulating evidences suggest that microRNAs (miRNAs) interact with their target genes and contribute to metabolic reprogramming in cancer cells. By integrating high-throughput screening data and the existing miRNA expression datasets, we explored the roles of candidate glycometabolism-regulating miRNAs in gastric cancer (GC). Subsequent investigation of the characterized miRNAs indicated that miR-129-5p inhibits glucose metabolism in GC cells. miRNA-129-5p directly targets the $3^{\prime}$-UTR of SLC2A3, thereby suppressing glucose consumption, lactate production, cellular ATP levels, and glucose uptake of GC cells. In addition, the PI3K-Akt and MAPK signaling pathways are involved in the effects of the miR-129-5p/SLC2A3 axis, regulating GC glucose metabolism and growth. These results reveal a novel role of the miR-129-5p/SLC2A3 axis in reprogramming the glycometabolism process in GC cells and indicate a potential therapeutic target for the treatment of this disease.

Keywords: miR-129-5p, gastric cancer, cancer metabolism, proliferation, SLC2A3

\section{INTRODUCTION}

Gastric cancer (GC) is the fifth most common malignancy in the world (Ferlay et al., 2015). In China, GC is the top three leading cause of cancer death among both men and women, causing approximately 679,100 new cancer cases and 498,000 deaths per year (Chen et al., 2016). Although the incidences and mortality trends for GC have declined in recent years (Siegel et al., 2014), the outcomes of this disease are still among the poorest of all solid-organ tumors, predominantly due to the frequent presence of advanced stage disease with lymphatic or distant metastasis. Current treatment strategies define curable GC as disease (stage 0-III) without distant metastasis (Shen et al., 2013), whereas stage IV disease remains incurable and carries a very poor prognosis despite

Abbreviations: 3'-UTR, 3'-untranslated region; GC, gastric cancer; GSEA, Gene Set Enrichment Analysis; SLC2A3, solute carrier family 2 member 3; TCGA, The Cancer Genome Atlas. 
the advent of molecularly targeted biological therapy and novel chemotherapy (Sonnenblick et al., 2015). As there are limited therapeutic approaches for treating advanced GC, it is urgent to elucidate new molecular mechanisms and develop more therapeutic targets for this lethal disease.

Cancer cells exhibit unrestrained growth, which are supported by metabolic adaptations that promote their survival. Aerobic glycolysis, or the Warburg effect (Warburg et al., 1927; Warburg, 1956a), a shift from oxidative phosphorylation to glycolysis and the concomitant accumulation of lactate byproducts in the surrounding microenvironment, represents the best-characterized alteration of tumor cell metabolism (Gillies et al., 2008; Cairns et al., 2011b). Altered glucose metabolism is characterized by increased glucose uptake, enhanced glycolysis, and dysregulated mitochondrial oxidative phosphorylation, as well as accumulated lactate production, events that are widespread in various cancer types (Warburg, 1956a,b; Shaw, 2006; Adekola et al., 2012). Recent studies have indicated that several key rate-limiting enzymes of glycometabolic pathways are dysregulated in GC cells, contributing to increased cell proliferation and metastasis (Cui et al., 2015; Jiang et al., 2015; Shiroki et al., 2017). MicroRNAs (miRNAs) are approximately 21-25 nucleotidelong, non-coding RNA molecules that negatively regulate gene translation by binding to the $3^{\prime}$-untranslated region ( $3^{\prime}$-UTR) of target messenger RNAs (mRNAs), causing either enhanced mRNA degradation or inhibited protein translation (Tay et al., 2008). miRNAs have critical effects that limit the glucose metabolism of cancer cells (Fong et al., 2015; Qiu et al., 2015), and emerging evidences suggest that aberrant miRNAs participate in the altered glycometabolism and pathogenesis of GC (Liu et al., 2016; Li C.Y. et al., 2016).

In this study, we explored potential glycometabolismregulating miRNAs in GC by integrating our previous highthroughput screening results and the existing GC miRNA expression datasets. miR-129-5p was identified as a candidate suppressor miRNA for glucose metabolism and cell proliferation, and SLC2A3 was a direct functional target gene of this miRNA in GC cells. The miR-129-5p/SLC2A3 axis may serve as a candidate therapeutic target for GC treatment.

\section{MATERIALS AND METHODS}

\section{Cell Culture}

The HEK 293T cells were cultured in Dulbecco's modified Eagle's medium (DMEM; Gibco, New York City, NY, United States), and SGC-7901 and MGC-803 cells were cultured in RPMI-1640 medium (HyClone, Beijing, China). The media were supplemented with $10 \%$ fetal bovine serum (Gibco), $100 \mathrm{IU} / \mathrm{mL}$ penicillin $\mathrm{G}$, and $100 \mu \mathrm{g} / \mathrm{mL}$ streptomycin sulfate (Sigma-Aldrich, St. Louis, MO, United States). PDGF-BB (Peprotech, Rocky Hill, CT, United States) was applied at the concentration of $50 \mathrm{ng} / \mathrm{mL}$ in RPMI-1640. Cells were cultured in a humidified $37^{\circ} \mathrm{C}$ incubator with $5 \% \mathrm{CO}_{2}$.

\section{Transfection of Oligonucleotides}

The miR-129-5p mimic (5'-CUUUUUGCGGUCUGGG CUUGC- $\left.3^{\prime}\right)$ and inhibitor (5'-GCAAGCCCAGACCGCA AAAAG- $3^{\prime}$ ) were designed and synthesized by Ambion (Austin, TX, United States). Three independent small interfering RNAs (siRNAs) targeting SLC2A3 were synthesized by Ribobio (Guangzhou, China), and the targeting sequences are as follows: 5'-GCTCTTTCCAATTTGGC TA-3', 5'-CCGACAGCCCATCATCATT- $3^{\prime}$ and $5^{\prime}$-GCTTCC TCATTACCTTCTT- $3^{\prime}$. Cells were transfected with the indicated oligonucleotides or a siRNA pool (3 siRNAs were mixed in an equimolar ratio) with final concentration of $50 \mathrm{nM}$, using Lipofectamine 2000 (Life Technologies, Darmstadt, Germany). At $48 \mathrm{~h}$ post-transfection, cells were harvested for further detection.

\section{Lactate Production and Glucose Consumption}

Cells were cultured in DMEM without phenol red (Gibco, New York City, NY, United States) for $15 \mathrm{~h}$, and then the culture medium was collected for lactate or glucose measurement. Quantification of lactate levels was performed utilizing Lactate Assay Kit (BioVision, Mountain View, CA, United States), and glucose levels were determined by Glucose Assay Kit (SigmaAldrich, Cat. No. GAHK20-1KT). All values were normalized by the corresponding total protein level (BCA Protein Assay Kit; Thermo Scientific, Waltham, MA, United States).

\section{Cellular ATP Levels}

The cellular levels of ATP were determined using CellTiterGlo ${ }^{\circledR}$ Luminescent Cell Viability Assay (Promega, Madison, WI, United States), following the manufacturer's instructions. All values were normalized by the corresponding total protein level.

\section{Glucose Uptake}

Cells were treated as indicated for $24 \mathrm{~h}$, then seeded in a 96-well plate (13,000 cells/well) and cultured for overnight. The culture medium was removed and cells were washed with PBS. Then, cells were incubated with fresh-prepared $500 \mu \mathrm{M} 2$-deoxyglucose (2-DG, $50 \mu \mathrm{L} /$ well) for $20 \mathrm{~min}$ at room temperature. The uptake process was stopped and neutralized, and luciferase activities were measured by Glucose Uptake-Glo Assay (Promega). Rate of glucose uptake was analyzed according to the manufacturer's instructions.

\section{Colony Formation Assays, Cell Proliferation, and EdU Incorporation Assay}

For colony formation assay, cells were plated in 6-well plate $(1,000$ cells/well $)$ and incubated 1 week, then fixed with $4 \%$ paraformaldehyde and stained with $1 \%$ crystal violet (Sigma-Aldrich). Megascopic cell colonies were counted. Cell proliferation was measured by Cell Counting Kit8 (CCK-8, Dojindo, Kumamoto, Japan), following the manufacturer's instructions. For EdU incorporation assay, cells were incubated with EdU (final concentration of 
$10 \mu \mathrm{mol} / \mathrm{L}$ ) for $2 \mathrm{~h}$ and then analyzed by Click-iT EdU Alexa Fluor ${ }^{\circledR}$ Imaging Kit (Molecular Probes, Eugene, OR, United States). Images were acquired using an Olympus DP71X microscope (Olympus, Tokyo, Japan), and EdU-positive cells were counted.

\section{Western Blot}

Proteins were separated on 7.5 or $10 \%$ sodium dodecyl sulfate-polyacrylamide gel and transferred to nitrocellulose membrane (Bio-Rad, Hercules, CA, United States). Then, the membrane was blocked with 5\% non-fat milk and incubated with indicated primary antibodies. The proteins were detected using enhanced chemiluminescence reagents (Thermo Scientific). The primary antibodies used were antiphospho-Akt (Ser473, \#9271; Cell Signaling Technology, Beverly, MA, United States), anti-Akt (\#9272, Cell Signaling Technology), anti-Phospho-Erk1/2 (Thr202/Tyr204, \#4370, Cell Signaling Technology), anti-p44/42 MAPK (Erk1/2, \#9102, Cell Signaling Technology), and anti- $\beta$-actin (60008-1-lg, Proteintech).

\section{RNA Extraction and Quantitative Real-Time Polymerase Chain Reaction (qPCR) Analysis}

Total RNA was extracted from cells with TRIzol ${ }^{\circledR}$ reagent (Invitrogen, Carlsbad, CA, United States). Reverse-transcribed complementary DNA was synthesized by PrimeScript ${ }^{\mathrm{TM}}$ RT Reagent Kit (TaKaRa, Tokyo, Japan). qPCR analyses were performed with SYBR Premix Ex Taq II Kit (TaKaRa, Tokyo, Japan). The detailed primer sequences are listed in Supplementary Table S1. Mature miR129-5p were quantified with specific primers and probes using TaqMan MicroRNA Assays (Applied Biosystems, Foster City, CA, United States), with U6 small nuclear RNA as internal control.

\section{Vector Construction}

The pri-miR-129 sequence was amplified from normal human genomic DNA and cloned into the pWPXL lentiviral vector (a generous gift from Dr. Didier Trono) to generate LentimiR-129-5p. pWPXL-SLC2A3 was constructed by inserting the open reading frame (ORF) of SLC2A3. The $3^{\prime}$-UTR sequence of SLC2A3 was amplified and inserted into the psiCHECK-2 vector (Promega, Madison, WI, United States). Detailed sequences of the primers and oligonucleotides are listed in Supplementary Table S1.

\section{Lentivirus Production and Transduction}

Lentiviral particles were harvested $48 \mathrm{~h}$ after Lenti-miR-129-5p or pWPXL-SLC2A3 co-transfection with the packaging plasmid psPAX2 and the VSV-G envelope plasmid pMD2.G (psPAX2 and pMD2.G were gifts from Dr. Didier Trono) into HEK 293T cells using Lipofectamine ${ }^{\circledR}$ 2000. SGC-7901 and MGC-803 cells were infected with the resultant recombinant lentivirus in the presence of $6 \mu \mathrm{g} / \mathrm{ml}$ polybrene (Sigma-Aldrich).

\section{Genome-Wide Transcriptional Profiling via the Complementary DNA (cDNA) Microarray}

The SGC-7901 and MGC-803 cells were transfected with miR129-5p mimics or control mimics. At $48 \mathrm{~h}$ post-transfection, cells were harvested for total RNA extraction. For microarray analysis, cells in TRIzol were shipped on dry ice to KangChen Bio-Tech (Shanghai, China) for analysis via the Agilent Whole Human Genome Oligo Microarray (one-color) platform. The RNA preparation and microarray hybridization were performed according to the manufacturer's instructions. Differentially downregulated genes were identified through fold-change filtering ( $\log _{2}$ Foldchange $<-1$ ). Pathway analysis and GO analysis were used to explore the roles of these differentially expressed genes.

\section{Luciferase Assays}

The HEK 293T cells were cultured in 96-well plates and cotransfected with $10 \mathrm{ng}$ of the psiCHECK-2-SLC2A3-3'-UTR vector and either $5 \mathrm{pmol}$ miR-129-5p mimics or control mimics. After $48 \mathrm{~h}$ of incubation, firefly and Renilla luciferase activities were measured using Dual-Luciferase Reporter Assay System (Promega).

\section{Statistical Analysis}

Results are presented as the means \pm standard error of the mean (SEM) from at least three independent experiments. Unless otherwise stated, differences between 2 groups or more than 2 groups were determined using Student's $t$-test or oneway analysis of variance (ANOVA), respectively, followed by Dunnett's multiple-comparisons test. $P$-values $<0.05$ were considered statistically significant. Statistical analyses were performed using GraphPad Prism for Windows, version 6.00 (GraphPad Software, San Diego, CA, United States).

\section{RESULTS}

\section{miR-129-5p Is a Repressor of Glucose Metabolism in GC Cells}

Lactate, the final product of aerobic glycolysis, is often overproduced in various types of tumors, which can be used to estimate the specific metabolomic characteristics and acidic microenvironment of tumor cells (Draoui and Feron, 2011). We previously identified 100 glycometabolismregulating miRNAs using a high-throughput lactate-production screening platform in HeLa cells (Guo W. et al., 2015). By summarizing previously reported miRNA expression datasets, 98 miRNAs were selected based on their frequent dysregulation in GC tissues (Supplementary Table S2). Candidate GC-glycometabolism-related miRNAs were defined as the intersection between the 98 dysregulated miRNAs in GC and the 100 candidate glycometabolismregulating miRNAs (Figure 1A). In total, seven candidate anti-metabolic miRNAs (miR-29c-3p, miR-34b-5p, miR-124-3p, miR-126-3p, miR-129-5p, miR-148a-3p, and miR-375) and 
A

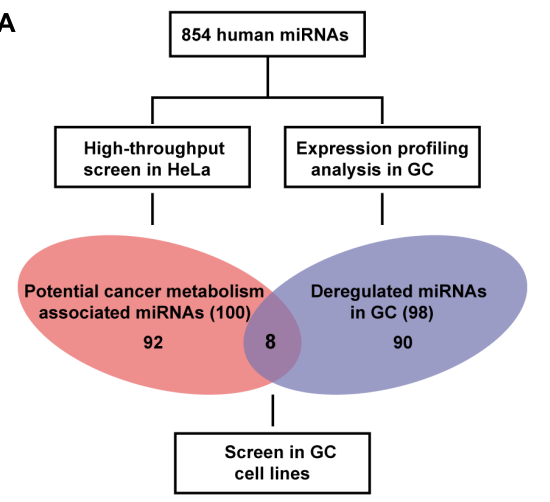

C

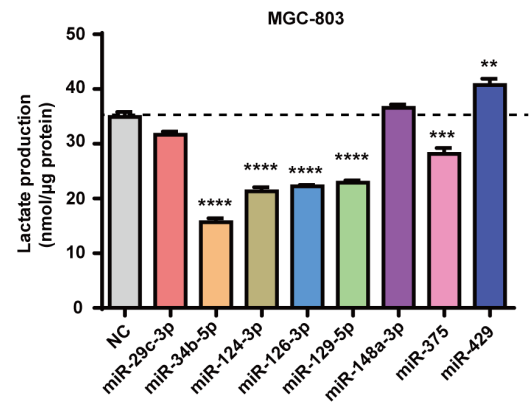

B

\begin{tabular}{ll}
\hline miRNA Name & Deregulation in GC \\
\hline miRNAs promote lactate production & \\
hsa-miR-429 & Up \\
miRNAs decrease lactate production & \\
hsa-miR-29c-3p & Down \\
hsa-miR-34b-5p & Down \\
hsa-miR-124-3p & Down \\
hsa-miR-126-3p & Down \\
hsa-miR-129-5p & Down \\
hsa-miR-148a-3p & Down \\
hsa-miR-375 & Down \\
\hline
\end{tabular}

D

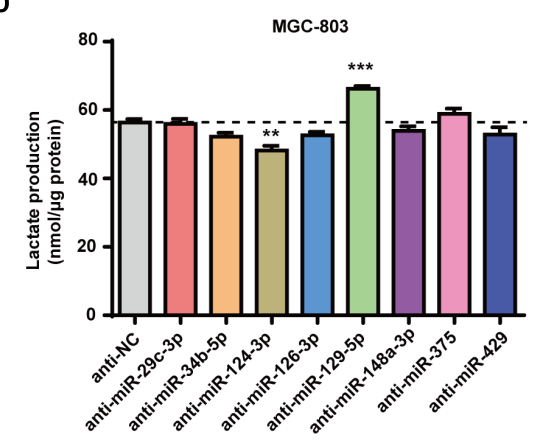

E

SGC-7901 MGC-803
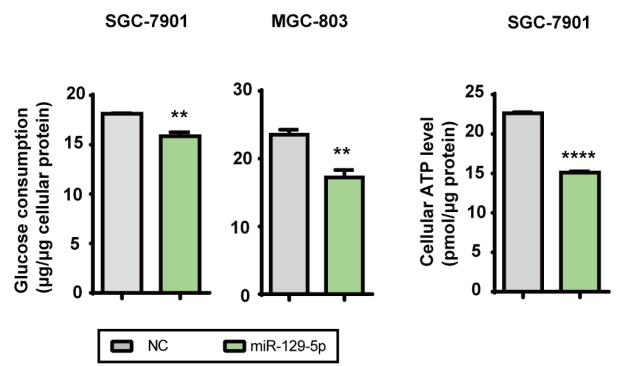

MGC-803

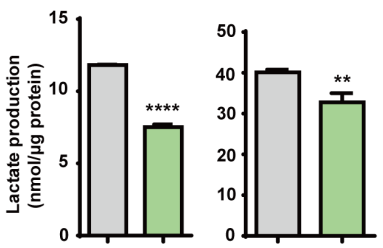

FIGURE 1 | MicroRNA-129-5p is a repressor of glucose metabolism in GC cells. (A) A schematic diagram of the protocol used to search for candidate metabolism-associated miRNAs in GC. (B) Eight miRNAs were identified as candidate glycometabolism-regulating miRNAs in GC. (C,D) Lactate production in MGC-803 cells after transfection of the indicated miRNAs (C) and corresponding inhibitors (D). (E) Lactate production, glucose consumption and cellular ATP levels in GC cells transfected with miR-129-5p mimic. Values are shown as the mean \pm standard error of the mean (SEM), $n=3$ in (C-E). ${ }^{* *} P<0.01 ;{ }^{* * *} P<0.001$; $* * * * P<0.0001$.

one candidate pro-metabolic miRNA (miR-429) emerged (Figure 1B).

To further confirm the specific effects of these eight miRNAs on GC glycometabolism, we evaluated lactate production of the MGC-803 cells that were transfected with indicated miRNA mimics or miRNA inhibitors. The results showed that miR129-5p dramatically repressed lactate production of MGC-803 cells (Figure 1C), whereas its inhibitor significantly elevated lactate production of MGC-803 cells (Figure 1D). Moreover, miR-129-5p mimics could reduce lactate production, glucose consumption and cellular ATP levels of SGC-7901 and MGC-803 cells (Figure 1E), indicating the potential role of miR-129-5p in GC glycometabolism. Taken together, these findings suggest that miR-129-5p inhibits glucose metabolism in GC cells.

\section{SLC2A3 Is the Direct Target of miR-129-5p in GC Cells}

To elucidate the mechanisms underlying the inhibitory effects of miR-129-5p on the glycometabolism of GC cells, we next identified its functional target genes. The glycometabolismrelated genes were clustered with the annotation of Gene Ontology Biological Function ${ }^{1}$. There were 29 glycometabolismrelated genes that were upregulated in the GSE13911 dataset ( $\log _{2}$ FoldChange $>1$, Supplementary Table S3), and 8 glycometabolism-related genes that were downregulated in miR-129-5p-treated MGC-803 cells ( $\log _{2}$ FoldChange $<-1$,

\footnotetext{
${ }^{1}$ http://geneontology.org/
} 
Supplementary Table S4). Then, these genes were assessed by TargetScan ${ }^{2}$ prediction and miRanda ${ }^{3}$ prediction, and SLC2A3 was identified as a potential target of miR-129-5p responsible for GC glycometabolism (Figure 2A). To further validate whether SLC2A3 could be directly regulated by miR-129-5p, the wild-type (WT) or mutant (MT) 3'-UTR of SLC2A3 was introduced into luciferase reporter plasmids (Figure 2B). There are two predicted miR-129-5p binding sites in the $3^{\prime}$-UTR of SLC2A3. miR-129-5p dramatically suppressed the luciferase activity of WT SLC2A3 3'UTR and MT2 SLC2A3 $3^{\prime}$-UTR and had a minor effect on MT1 SLC2A3 3'-UTR, but did not affect MT (1+2) SLC2A3 3'-UTR, suggesting that miR-129-5p predominantly binds to the first predicted site (1804-1825 nt) of SLC2A3 3'-UTR (Figure 2C). Moreover, miR-129-5p mimic significantly reduced the mRNA and protein levels of SLC2A3 in GC cells (Figure 2D).

\section{The miR-129-5p/SLC2A3 Axis Regulates Glucose Metabolism in GC Cells}

Given that miR-129-5p represses glucose metabolism in GC cells, we next investigated the possible roles of its target gene SLC2A3 in GC glucose metabolism. Silencing of the endogenous SLC2A3 with siRNAs resulted in the dramatic suppression

\footnotetext{
${ }^{2}$ http://www.targetscan.org

${ }^{3}$ http://www.microrna.org
}

of the glucose consumption, lactate production, cellular ATP levels, and glucose uptake of GC cells (Figure $\mathbf{3 A}$ and Supplementary Figure S1A), which phenocopied the inhibitory effect of miR-129-5p on GC glycometabolism. Furthermore, we established SGC-7901 and MGC-803 cells with stable miR-129-5p overexpression via a lentivirus system (designated as Lenti-miR129-5p, Supplementary Figure S1B), and constructed a lentivirus plasmid containing an SLC2A3 cDNA sequence without the $3^{\prime}$ UTR to reintroduce SLC2A3 into GC cells that overexpressed miR-129-5p (Supplementary Figure S1C). As expected, miR129-5p overexpression decreased the lactate secretion, glucose consumption, cellular ATP levels, and glucose uptake of SGC7901 and MGC-803 cells (Figure 3B), similar to the inhibitory effect of the specific mimics. Moreover, restoration of SLC2A3 in GC cells significantly abolished the miR-129-5p-induced suppression of lactate excretion, glucose consumption, cellular ATP levels, and glucose uptake (Figure 3B). These results suggest that miR-129-5p may regulate glycometabolism through SLC2A3 expression in GC cells.

\section{The miR-129-5p/SLC2A3 Axis Regulates the Proliferation of GC Cells}

Over the recent decade, advanced studies of cancer metabolism have broadened our understanding of how disturbed glucose metabolism can be involved in carcinogenesis
A
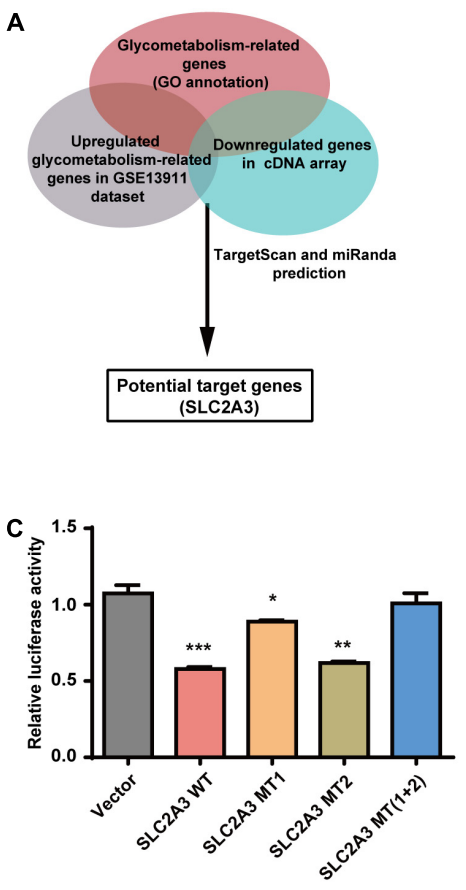

B $\begin{array}{ll}\text { SLC2A3 3'UTR WT (1804-1825) } & \text { 5'... GTCTCTACAAAAAUGCAAAAAU ....3' } \\ \text { III I III I I } \\ \text { hsa-miR-129-5p } & \text { 3'... CGUUCGGGUCUGGCGUUUUC } \\ \text { SLC2A3 3'UTR MT1 (1804-1825) } & \text { 5'... GTCTCTACAAAAAUCGUUUUUU ...3' }\end{array}$

SLC2A3 3'UTR WT (1959-1979) 5'... GCGAGACTCCGTCTCAAAAAA ...3' hsa-miR-129-5p $\quad 3^{\prime} . .$. CGUUCGGGUCUGGCGUUUUUC SLC2A3 3'UTR MT2 (1959-1979) 5'... GCGAGACTCCGTCTUCCGCUA ...3'

FIGURE 2 | SLC2A3 is the direct target of miR-129-5p in GC cells. (A) Schematic representation of the strategy used to identify candidate target genes of miR-129-5p. (B) Diagram of putative miR-129-5p binding sites in the $3^{\prime}-U T R$ of SLC2A3. The mutant sequences of SLC2A3 $3^{\prime}-U T R$ used in the luciferase reporter constructs are indicated in red. (C) Relative activities of luciferase reporters containing SLC2A3 3'-UTR variants co-transfected with miR-129-5p or negative control mimics in HEK 293T cells. (D) SLC2A3 mRNA and protein levels in GC cells transfected with miR-129-5p mimics. Values are shown as the mean \pm SEM, $n=3$ in (C,D). ${ }^{*} P<0.05 ;{ }^{* *} P<0.01 ; * * P<0.001$. 
A

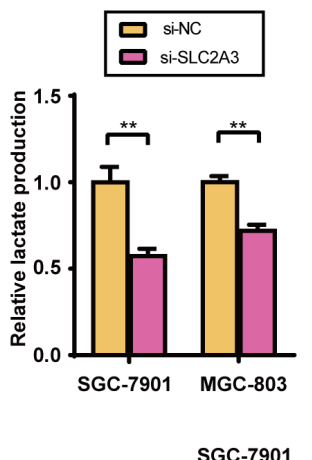

B
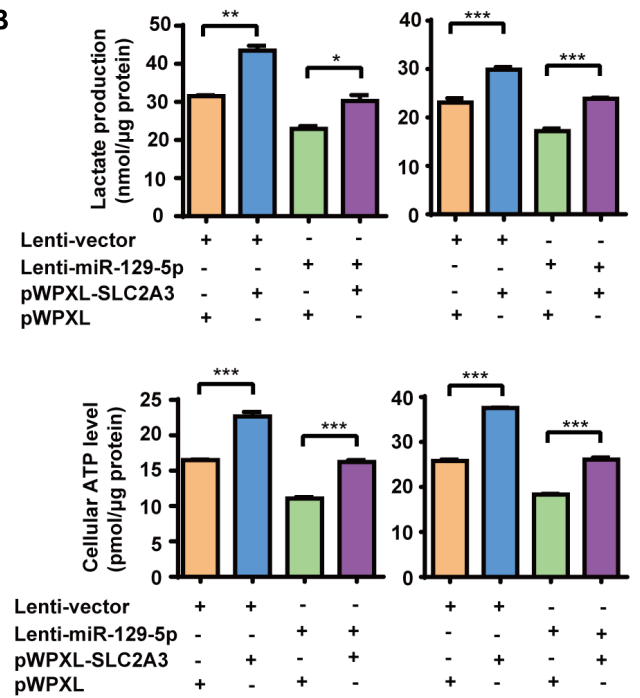
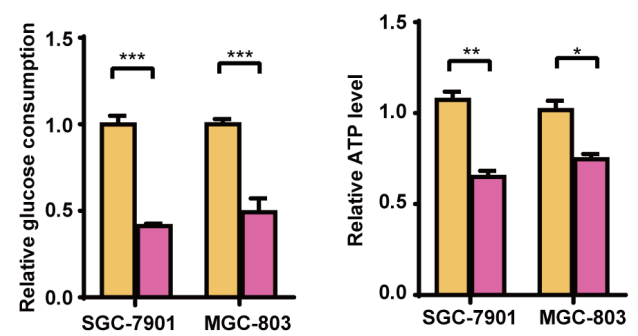

SGC-7901
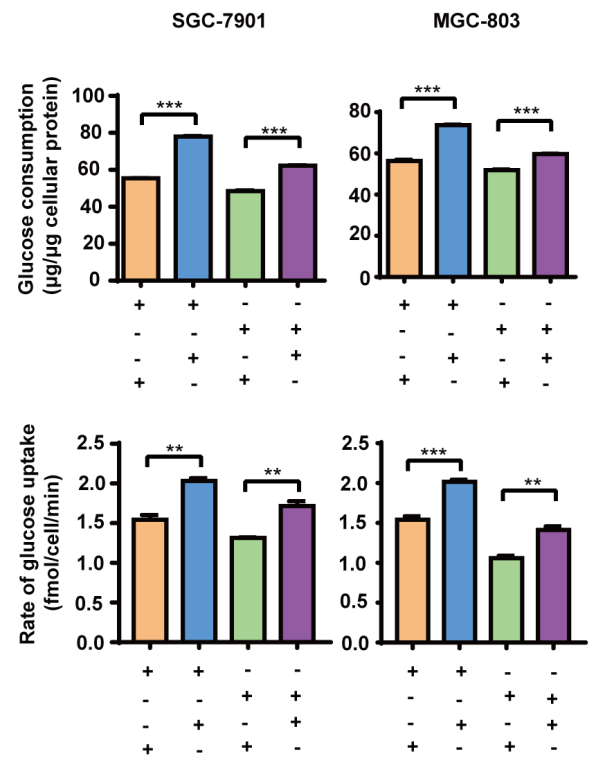

FIGURE 3 | The miR-129-5p/SLC2A3 axis regulates glucose metabolism in GC cells. (A) SLC2A3 knockdown suppressed lactate production, glucose consumption, cellular ATP levels and glucose uptake in GC cells. (B) The restoration of SLC2A3 protein expression in GC cells significantly abolished the suppressive effects of miR-129-5p on lactate excretion, glucose consumption, cellular ATP levels and glucose uptake in GC cells. Values are shown as the mean \pm SEM, $n=3$. ${ }^{*} P<0.05 ;{ }^{* *} P<0.01 ;{ }^{* * *} P<0.001$

(Ward and Thompson, 2012). miR-129-5p was previously identified as a glycometabolism-related miRNA in GC, then we further characterized its effects on the proliferation of GC cells. EdU incorporation assays showed that miR-129-5p mimic treatment significantly inhibited the proliferation of SGC-7901 and MGC-803 cells (Figure 4A). Moreover, miR-129-5p mimic treatment suppressed the colony formation ability of GC cells (Figure 4B). These results demonstrated that miR-129-5p might act as a suppressor miRNA in GC carcinogenesis. Indeed, miR-129-5p was significantly downregulated in GC tissues in the TCGA cohort (Supplementary Figure S2), compared with that in non-tumor tissues.

Regarding the function of miR-129-5p/SLC2A3 axis in regulating GC glycometabolism, we then examined whether this axis might contribute to the proliferation of GC cells. As shown in Figure 4C, Lenti-129-5p treatment significantly inhibited the colony formation abilities of SGC7901 and MGC-803 cells, whereas SLC2A3 overexpression dramatically enhanced the colony formation abilities of these cells. Importantly, ectopic expression of SLC2A3 overcame the anti-proliferation effects of miR-129-5p in GC cells (Figure 4C), indicating that targeting SLC2A3 is an important mechanism for the anti-proliferation function of miR-129-5p.

\section{miR-129-5p Reprograms Gene Expression Profiling in GC Cells}

To identify the molecular processes and signaling pathways underlying the suppressor activity of miR-129-5p in gastric glycometabolism and carcinogenesis, the gene expression profiling of miR-129-5p-treated MGC-803 cells was analyzed by cDNA microarrays. Functional annotation revealed that signaling pathway gene sets that primarily affected by miR129-5p include PI3K-Akt signaling pathway, MAPK signaling pathway and Hippo signaling pathways, resulting in the remarkable changes in protein processing in the endoplasmic reticulum and proteoglycans process in cancer (Figure 5A). The cDNA microarray also confirmed that genes in the top-scoring processes, such as those in PI3K-Akt signaling pathway and MAPK signaling pathway (Figure 5B), could 


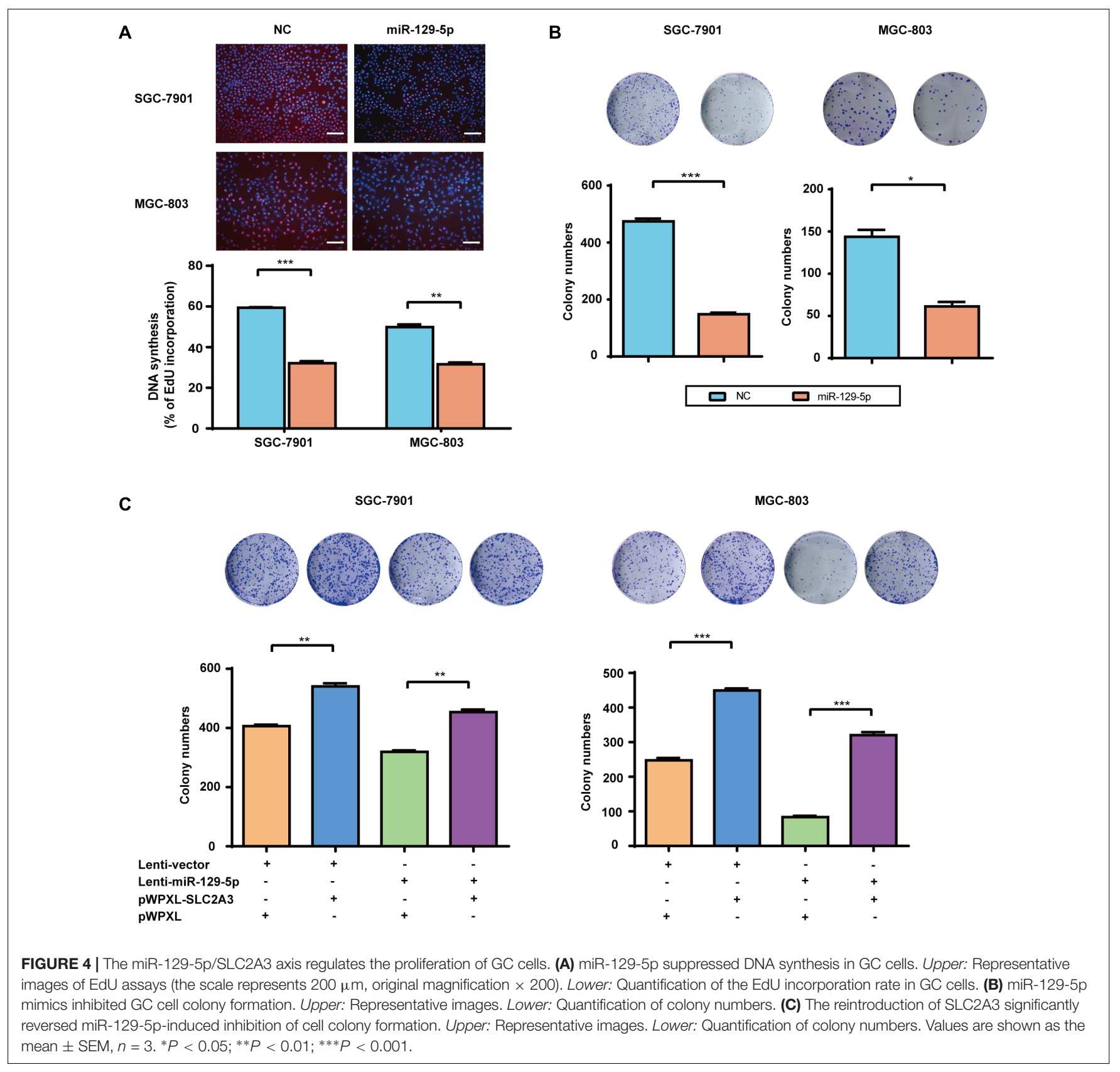

be regulated by miR-129-5p in MGC-803 cells (Figure 5C). Furthermore, miR-129-5p could inhibit the phosphorylation levels of Akt and Erk1/2 in GC cells without obvious changes in the total levels of these proteins, and SLC2A3 knockdown by specific siRNAs suppressed Akt and Erk1/2 phosphorylation (Figure 5D), similar to the effects of miR-129-5p overexpression in GC cells. Moreover, when SGC-7901 and MGC-803 cells were treated with only PDGF$\mathrm{BB}$, introduction of miR-129-5p in these cells remarkably abolished the promoting effects of PDGF-BB on GC cell proliferation (Figure 5E). These results suggest that the miR-129-5p/SLC2A3 axis exerts its biological activities on glycometabolism and carcinogenesis primarily through modulating the PI3K-Akt and MAPK signaling pathways in GC cells.

\section{DISCUSSION}

Emerging evidences suggest that aberrant miRNAs participate in the pathogenesis of GC (Guo L.L. et al., 2015). Recently, several miRNAs, such as miR-let-7a, miR-148b and miR-181b, were identified as regulators of glycolysis and the citric acid cycle in GC (Tang et al., 2016; Li L.Q. et al., 2016; Ding et al., 2017). We previously identified 100 glycometabolismregulating miRNAs from an 854-miRNA library using a 


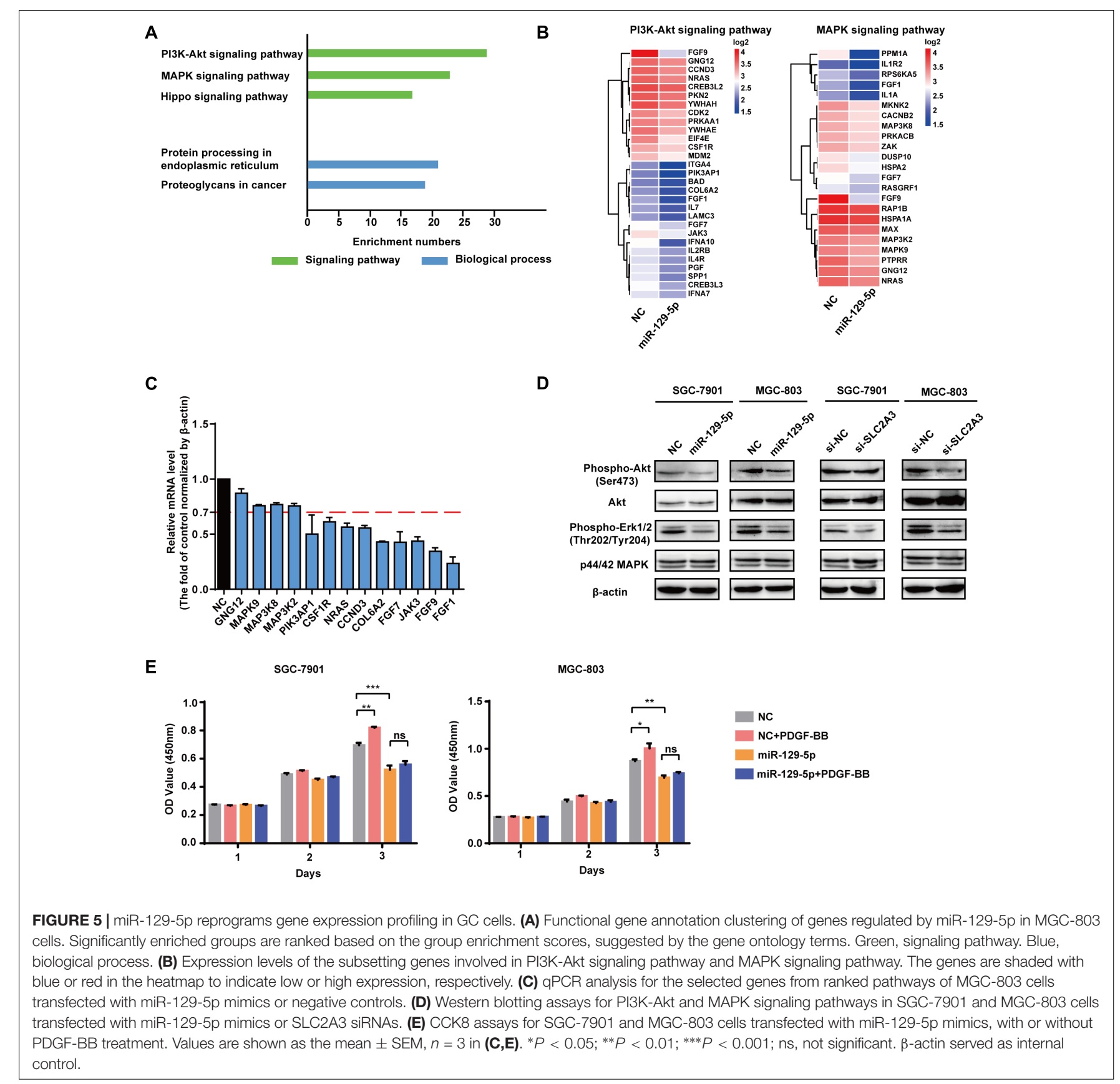

high-content screening procedure (Guo W. et al., 2015). In the present study, we demonstrated that miR-129-5p could dramatically repress lactate production, glucose consumption, cellular ATP levels, and glucose uptake in GC cells. miR-129 was previously implicated in gastrointestinal cancer (Fesler et al., 2014), due to targeting important genes that are associated with tumorigenesis, disease progression, cell cycle, cell motility, and chemoresistance. The level miR-129-5p was found to be significantly decreased in GC and positively associated with overall survival of GC patient (Li C.Y. et al., 2016). In addition to targeting coding genes, miR-129-5p contributed to lncRNA-AC130710 upregulation in GC tissues (Xu et al.,
2014). Hypermethylation of a miR-129-5p CpG island might play important roles in the development of GC chemoresistance (Wu et al., 2014). As expected, ectopic expression of miR-129$5 p$ significantly inhibited the colony formation abilities and the growth of GC cells. Our findings highlight an additional mechanism for miR-129-5p-inhibited cell growth, which is mediated by the regulation of glucose metabolism in GC cells.

Tumor cells increase their glucose consumption to generate the necessary biomass for their proliferation (Cairns et al., 2011a), and they upregulate facilitative glucose transporter (GLUT) proteins to achieve sufficient glucose uptake (Barron et al., 2016). 
GLUT proteins, encoded by the $S L C 2$ genes, are members of the major facilitator superfamily of membrane transporters (Mueckler and Thorens, 2013), and responsible for the first step of cellular glucose utilization by facilitative diffusion of glucose (Masin et al., 2014). SLC2A3 (GLUT3), a transporter with a high affinity for glucose (Km approximately $1.5 \mathrm{mM}$ ) and the highest calculated glucose turnover rate, transports glucose across the cell membrane in an energy-independent manner (Rodriguez-Enriquez et al., 2009). Including GC, positive staining results for SLC2A3 have been detected in several malignant tumor tissues (Younes et al., 1997), suggesting that SLC2A3 may participate in facilitating glucose uptake in the tumors with intense glucose requirements. Previous studies have shown that SLC2A3 could be directly regulated by miR106a in glioblastoma (Dai et al., 2013), as well as by miR195-5p in bladder cancer (Fei et al., 2012). In this study, we demonstrate that SLC2A3 is a direct functional target of miR129-5p in GC cells and the miR-129-5p/SLC2A3 axis plays important roles in the reprogramming of glycometabolism for tumor bioenergetics and biosynthesis, thus influencing GC cell growth.

Our study further shows that PI3K-Akt signaling pathway and MAPK signaling pathway are involved in mediating the effects of miR-129-5p in GC cells. Gene Set Enrichment Analysis (GSEA) revealed that these two signaling pathways were the topscoring processes affected by miR-129-5p in GC cells. As Akt and MAPK are pivotal growth-promoting signaling modulators (Wilhelm et al., 2004; Garcia et al., 2006), it is not surprising that they contribute to the proliferation of GC cells as downstream effectors of many stimuli (Wang et al., 2012; Ye et al., 2016). However, the extensive interplay between these pathways and miRNAs, that facilitates the Warburg effect and cell growth in GC cells, is still poorly understood. Our results revealed that the glycometabolism-related miR-129-5p/SLC2A3 axis could significantly affect the phosphorylation of Akt and ERK1/2, and miR-129-5p could erase the promoting effects of PDGF-BB on GC cell proliferation, indicating that PI3K-Akt and MAPK signaling pathways may be implicated in the regulated GC glycometabolism and cell growth by the miR-129-5p/SLC2A3 axis.

\section{REFERENCES}

Adekola, K., Rosen, S. T., and Shanmugam, M. (2012). Glucose transporters in cancer metabolism. Curr. Opin. Oncol. 24, 650-654. doi: 10.1097/CCO. 0b013e328356da72

Barron, C. C., Bilan, P. J., Tsakiridis, T., and Tsiani, E. (2016). Facilitative glucose transporters: implications for cancer detection, prognosis and treatment. Metabolism 65, 124-139. doi: 10.1016/j.metabol.2015. 10.007

Cairns, R. A., Harris, I. S., and Mak, T. W. (2011a). Regulation of cancer cell metabolism. Nat. Rev. Cancer 11, 85-95. doi: 10.1038/nrc2981

Cairns, R. A., Harris, I., McCracken, S., and Mak, T. W. (2011b). Cancer cell metabolism. Cold Spring Harb. Symp. Quant. Biol. 76, 299-311. doi: 10.1101/ sqb.2011.76.012856

Chen, W., Zheng, R., Baade, P. D., Zhang, S., Zeng, H., Bray, F., et al. (2016). Cancer statistics in China, 2015. CA Cancer J. Clin. 66, 115-132. doi: 10.3322/ caac. 21338

\section{CONCLUSION}

We demonstrate that miR-129-5p is a vital regulator of glucose metabolism and cell proliferation in GC cells, and SLC2A3 is the direct functional target of this miRNA. The newly identified miR-129-5p/SLC2A3 axis plays an essential role in GC glucose metabolism and cell growth through the involvement of the PI3K-Akt and MAPK pathways. Our observations provide new insights into the pathogenesis of GC and suggest a novel potential therapeutic target for the treatment of this disease.

\section{ETHICS STATEMENT}

Before the experiments, all procedures were approved by the ethical committee of the Fudan University.

\section{AUTHOR CONTRIBUTIONS}

$\mathrm{XH}, \mathrm{YZ}, \mathrm{DC}$, and HW conceived of the presented idea. DC and HW carried out the experiment. DC wrote the manuscript with support from XH, YZ, and HW. DC, YZ, HW, SL and $\mathrm{SH}$ analyzed the data. All authors discussed the results and contributed to the final manuscript.

\section{FUNDING}

This work was supported by grants from the National Natural Science Foundation of China (81472565, 81672727).

\section{SUPPLEMENTARY MATERIAL}

The Supplementary Material for this article can be found online at: https://www.frontiersin.org/articles/10.3389/fphar. 2018.00502/full\#supplementary-material

Cui, Y., Qin, L., Wu, J., Qu, X., Hou, C., Sun, W., et al. (2015). SIRT3 enhances glycolysis and proliferation in SIRT3-expressing gastric cancer Cells. PLoS One 10:e0129834. doi: 10.1371/journal.pone.0129834

Dai, D. W., Lu, Q., Wang, L. X., Zhao, W. Y., Cao, Y. Q., Li, Y. N., et al. (2013). Decreased miR-106a inhibits glioma cell glucose uptake and proliferation by targeting SLC2A3 in GBM. BMC Cancer 13:478. doi: 10.1186/1471-2407$13-478$

Ding, X., Liu, J., Liu, T., Ma, Z., Wen, D., and Zhu, J. (2017). miR-148b inhibits glycolysis in gastric cancer through targeting SLC2A1. Cancer Med. 6, 1301-1310. doi: 10.1002/cam4.1008

Draoui, N., and Feron, O. (2011). Lactate shuttles at a glance: from physiological paradigms to anti-cancer treatments. Dis. Model Mech. 4, 727-732. doi: 10.1242/dmm.007724

Fei, X., Qi, M., Wu, B., Song, Y., Wang, Y., and Li, T. (2012). MicroRNA-195-5p suppresses glucose uptake and proliferation of human bladder cancer T24 cells by regulating GLUT3 expression. FEBS Lett. 586, 392-397. doi: 10.1016/j.febslet. 2012.01.006 
Ferlay, J., Soerjomataram, I., Dikshit, R., Eser, S., Mathers, C., Rebelo, M., et al. (2015). Cancer incidence and mortality worldwide: sources, methods and major patterns in GLOBOCAN 2012. Int. J. Cancer 136, E359-E386. doi: 10.1002/ijc. 29210

Fesler, A., Zhai, H., and Ju, J. (2014). miR-129 as a novel therapeutic target and biomarker in gastrointestinal cancer. Onco Targets Ther. 7, 1481-1485. doi: 10.2147/OTT.S65548

Fong, M. Y., Zhou, W., Liu, L., Alontaga, A. Y., Chandra, M., Ashby, J., et al. (2015). Breast-cancer-secreted miR-122 reprograms glucose metabolism in premetastatic niche to promote metastasis. Nat. Cell Biol. 17, 183-194. doi: $10.1038 /$ ncb3094

Garcia, Z., Kumar, A., Marques, M., Cortes, I., and Carrera, A. C. (2006). Phosphoinositide 3-kinase controls early and late events in mammalian cell division. EMBO J. 25, 655-661. doi: 10.1038/sj.emboj.7600967

Gillies, R. J., Robey, I., and Gatenby, R. A. (2008). Causes and consequences of increased glucose metabolism of cancers. J. Nucl. Med. 49(Suppl. 2), 24S-42S. doi: 10.2967/jnumed.107.047258

Guo, L. L., Song, C. H., Wang, P., Dai, L. P., Zhang, J. Y., and Wang, K. J. (2015). Competing endogenous RNA networks and gastric cancer. World J. Gastroenterol. 21, 11680-11687. doi: 10.3748/wjg.v21.i41.11680

Guo, W., Qiu, Z., Wang, Z., Wang, Q., Tan, N., Chen, T., et al. (2015). MiR-199a$5 \mathrm{p}$ is negatively associated with malignancies and regulates glycolysis and lactate production by targeting hexokinase 2 in liver cancer. Hepatology 62, 1132-1144. doi: 10.1002/hep.27929

Jiang, W., Zhou, F., Li, N., Li, Q., and Wang, L. (2015). FOXM1-LDHA signaling promoted gastric cancer glycolytic phenotype and progression. Int. J. Clin. Exp. Pathol. 8, 6756-6763.

Li, C. Y., Liang, G. Y., Yao, W. Z., Sui, J., Shen, X., Zhang, Y. Q., et al. (2016). Identification and functional characterization of microRNAs reveal a potential role in gastric cancer progression. Clin. Transl. Oncol. 19, 162-172. doi: 10.1007/ s12094-016-1516-y

Li, L. Q., Yang, Y., Chen, H., Zhang, L., Pan, D., and Xie, W. J. (2016). MicroRNA$181 \mathrm{~b}$ inhibits glycolysis in gastric cancer cells via targeting hexokinase 2 gene. Cancer Biomark. 17, 75-81. doi: 10.3233/CBM-160619

Liu, L., Wang, Y., Bai, R., Yang, K., and Tian, Z. (2016). MiR-186 inhibited aerobic glycolysis in gastric cancer via HIF-lalpha regulation. Oncogenesis 5:e224. doi: 10.1038 /oncsis. 2016.35

Masin, M., Vazquez, J., Rossi, S., Groeneveld, S., Samson, N., Schwalie, P. C., et al. (2014). GLUT3 is induced during epithelial-mesenchymal transition and promotes tumor cell proliferation in non-small cell lung cancer. Cancer Metab. 2:11. doi: 10.1186/2049-3002-2-11

Mueckler, M., and Thorens, B. (2013). The SLC2 (GLUT) family of membrane transporters. Mol. Aspects Med. 34, 121-138. doi: 10.1016/j.mam.2012.07.001

Qiu, Z., Guo, W., Wang, Q., Chen, Z., Huang, S., Zhao, F., et al. (2015). MicroRNA124 reduces the pentose phosphate pathway and proliferation by targeting PRPS1 and RPIA mRNAs in human colorectal cancer cells. Gastroenterology 149, 1587.e11-1598.e11. doi: 10.1053/j.gastro.2015.07.050

Rodriguez-Enriquez, S., Marin-Hernandez, A., Gallardo-Perez, J. C., and MorenoSanchez, R. (2009). Kinetics of transport and phosphorylation of glucose in cancer cells. J. Cell. Physiol. 221, 552-559. doi: 10.1002/jcp. 21885

Shaw, R. J. (2006). Glucose metabolism and cancer. Curr. Opin. Cell Biol. 18, 598-608. doi: 10.1016/j.ceb.2006.10.005

Shen, L., Shan, Y. S., Hu, H. M., Price, T. J., Sirohi, B., Yeh, K. H., et al. (2013). Management of gastric cancer in Asia: resource-stratified guidelines. Lancet Oncol. 14, e535-e547. doi: 10.1016/S1470-2045(13)70436-4

Shiroki, T., Yokoyama, M., Tanuma, N., Maejima, R., Tamai, K., Yamaguchi, K., et al. (2017). Enhanced expression of the M2 isoform of pyruvate kinase is involved in gastric cancer development by regulating cancer-specific metabolism. Cancer Sci. 108, 931-940. doi: 10.1111/cas.13211
Siegel, R., Ma, J., Zou, Z., and Jemal, A. (2014). Cancer statistics, 2014. CA Cancer J. Clin. 64, 9-29. doi: 10.3322/caac.21208

Sonnenblick, A., de Azambuja, E., Azim, H. A. Jr., and Piccart, M. (2015). An update on PARP inhibitors-moving to the adjuvant setting. Nat. Rev. Clin. Oncol. 12, 27-41. doi: 10.1038/nrclinonc.2014.163

Tang, R., Yang, C., Ma, X., Wang, Y., Luo, D., Huang, C., et al. (2016). MiRlet-7a inhibits cell proliferation, migration, and invasion by down-regulating PKM2 in gastric cancer. Oncotarget 7, 5972-5984. doi: 10.18632/oncotarget. 6821

Tay, Y., Zhang, J., Thomson, A. M., Lim, B., and Rigoutsos, I. (2008). MicroRNAs to Nanog, Oct4 and Sox 2 coding regions modulate embryonic stem cell differentiation. Nature 455, 1124-1128. doi: 10.1038/nature 07299

Wang, J., Gui, Z., Deng, L., Sun, M., Guo, R., Zhang, W., et al. (2012). c-Met upregulates aquaporin 3 expression in human gastric carcinoma cells via the ERK signalling pathway. Cancer Lett. 319, 109-117. doi: 10.1016/j.canlet.2011. 12.040

Warburg, O. (1956a). On respiratory impairment in cancer cells. Science 124, 269-270.

Warburg, O. (1956b). On the origin of cancer cells. Science 123, 309-314. doi: 10.1126/science.123.3191.309

Warburg, O., Wind, F., and Negelein, E. (1927). The metabolism of tumors in the body. J. Gen. Physiol. 8, 519-530. doi: 10.1085/jgp.8.6.519

Ward, P. S., and Thompson, C. B. (2012). Metabolic reprogramming: a cancer hallmark even Warburg did not anticipate. Cancer Cell 21, 297-308. doi: 10.1016/j.ccr.2012.02.014

Wilhelm, S. M., Carter, C., Tang, L., Wilkie, D., McNabola, A., Rong, H., et al. (2004). BAY 43-9006 exhibits broad spectrum oral antitumor activity and targets the RAF/MEK/ERK pathway and receptor tyrosine kinases involved in tumor progression and angiogenesis. Cancer Res. 64, 7099-7109. doi: 10.1158/ 0008-5472.CAN-04- 1443

Wu, Q., Yang, Z., Xia, L., Nie, Y., Wu, K., Shi, Y., et al. (2014). Methylation of miR-129-5p CpG island modulates multi-drug resistance in gastric cancer by targeting ABC transporters. Oncotarget 5, 11552-11563. doi: 10.18632/ oncotarget. 2594

Xu, C., Shao, Y., Xia, T., Yang, Y., Dai, J., Luo, L., et al. (2014). IncRNA-AC130710 targeting by miR-129-5p is upregulated in gastric cancer and associates with poor prognosis. Tumour Biol. 35, 9701-9706. doi: 10.1007/s13277-014-2274-5

Ye, Y., Ge, Y. M., Xiao, M. M., Guo, L. M., Li, Q., Hao, J. Q., et al. (2016). Suppression of SHIP2 contributes to tumorigenesis and proliferation of gastric cancer cells via activation of Akt. J. Gastroenterol. 51, 230-240. doi: 10.1007/ s00535-015-1101-0

Younes, M., Lechago, L. V., Somoano, J. R., Mosharaf, M., and Lechago, J. (1997). Immunohistochemical detection of Glut3 in human tumors and normal tissues. Anticancer Res. 17, 2747-2750.

Conflict of Interest Statement: The authors declare that the research was conducted in the absence of any commercial or financial relationships that could be construed as a potential conflict of interest.

The reviewer XT and handling Editor declared their shared affiliation.

Copyright (C) 2018 Chen, Wang, Chen, Li, Li, Hu, Huang, Zhao and He. This is an open-access article distributed under the terms of the Creative Commons Attribution License (CC BY). The use, distribution or reproduction in other forums is permitted, provided the original author(s) and the copyright owner are credited and that the original publication in this journal is cited, in accordance with accepted academic practice. No use, distribution or reproduction is permitted which does not comply with these terms. 